论文

\title{
广角 $\mathbf{X}$ 射线散射实验的散射角度修正
}

\author{
赵镍，边风刚，王玉柱，杨春明，周平，王劼 ${ }^{*}$
}

上海同步辐射光源, 中国科学院上海应用物理研究所, 上海 201204

*联系人, E-mail: wangjie@ sinap.ac.cn

收稿日期: 2014-04-23; 接受日期: 2014-06-18

上海光源线站运行研究项目 (“连续 SAXS-WAXS 散射矢量的测量”)和国家重点基础研究发展计划(编号: 2010CB934501, 2011CB2606104, 2011CB11104, 2011CB605604)资助项目

摘要在同步辐射小角与广角联用 X 射线散射实验中由于原位实验设备的引入, 探测器所获得的散射 角并非真实的散射角，因此需要对探测值进行修正以获得真实的散射角. 校正过程需要解决两个问题: 一是要建立一个方程来完整描述实验中可能产生角度偏差的各种因素; 另一个问题是寻找探测值和真实 散射角的对应关系. 本文以一维弧形探测器为原型建立了一种通用构造校正方程的方法，首先建立以样 品中心为原点的几何模型方程，然后根据精度要求对方程进行泰勒近似，获得了简化的校正方程．通过 模拟不同实验条件下的真实散射角与探测值，证实了校正方程的可行性，同时模拟结果也可以应用于解 决寻峰的问题, 最后选取 $\mathrm{LaB}_{6}, \mathrm{CeO}_{2}, \mathrm{Y}_{2} \mathrm{O}_{3}$ 三种标准样品在不同实验条件下对校正精度进行了验证. 根 据探测器偏离样品中心的距离可分为三种模式(模式一<模式二<模式三), 模式二、三的校正结果与理论 分析相吻合，而模式一由于角度分辨较小以及视差效应等造成校正精度略大于理论预测值. 这一方法同 样适应于一维线性探测器、二维面形探测器等广角探测器.

关键词同步辐射, $X$ 射线散射, 广角衍射，角度修正

PACS: 06.20.fb, 07.85.Qe, 78.70.Ck, 07.05.Tp

doi: 10.1360/SSPMA2014-00106

\section{1 引言}

小角 $\mathrm{X}$ 射线散射 $(\mathrm{SAXS})^{[1]}$ 是纳米材料领域中一 种重要的分析技术, 具有制样简单, 对样品的形态要 求低, 且在测试过程不破坏样品等特点, 因此广泛应 用于高分子材料 ${ }^{[2,3]}$ 、合金 ${ }^{[4,5]}$ 、生物大分子溶液 ${ }^{[6]}$ 等 多种体系的纳米尺度范围内的结构表征. 然而基于 常规 X 射线源的 SAXS 并不能实现对材料动态过程
的观察, 而且要求材料中的纳米结构具有较大的电 子密度差, 因此对于弱散射体系往往难以获得高信 噪比的数据. 随着第三代同步辐射技术的引入, 光通 量的大幅提升使得 SAXS 技术不仅可以实现对弱散 射体系的快速测量, 而且多种新型 SAXS 实验方法得 以发展起来，其中较为突出的是基于同步辐射的时 间分辨小角和广角联用 $\mathrm{X}$ 射线散射技术(SR-SWA XS), 它可以用于从原子到大分子尺度范围内结构的

引用格式: 赵镍, 边风刚, 王玉柱, 等. 广角 X 射线散射实验的散射角度修正. 中国科学: 物理学 力学 天文学, 2015, 45: 017001 Zhao N, Bian F G, Wang Y Z, et al. Calibrating scattering angle in wide angle X-ray scattering experiment (in Chinese). Sci Sin-Phys Mech Astron, 2015, 45: 017001, doi: 10.1360/SSPMA2014-00106 
表征，时间分辨则使针对材料内部纳米结构尺度的 动力学研究得以开展, 例如应用 SWAXS 研究聚合物 拉伸诱导结晶过程和蛋白质溶液中生物大分子随环 境的改变而发生的形态变化, 这一实验方法得到了 世界范围内各个 SAXS 实验站的重视, 自 20 世纪 90 年代以来, 世界范围内先后发展 SWAXS 实验方法并 正在运行的实验站主要有: EMBL 的 $\mathrm{X} 33(\mathrm{D} 1 / 2)^{[7]}$ 束 线站, SSRL 的 BL4- $2^{[8]}$ 束线站, NSLS 的 BNL, X27C 束线站 ${ }^{[9]}$, ESRF 的 ID02 $2^{[10]}$, ID13 ${ }^{[11]}$, DUBBLE ${ }^{[12]}$ 束线 站, Elettra 的 BL5.2 $2^{[13]}$ 束线站, Spring 的 BL-45XU ${ }^{[14]}$ 束线站, 台湾 NSRRC 的 BL17B $3^{[15]}$ 和 BL23 $\mathrm{A}^{[16]}$ 实验 站, BESSY II 的 $\mu$-Spot 实验站 ${ }^{[17]}$, DELTA 的 BL $9^{[18]}$ 束线站, ESRF 的 $\mathrm{ID} 15 \mathrm{~A}^{[19]}$. 上海同步辐射光源的 BL16B 光束线站最近发展了 SWAXS 实验方法, 可以 实现聚合物拉伸过程和温控过程中材料的结构演变 等动态过程的研究 ${ }^{[20]}$.

SWAXS 实验方法是在常规 SAXS 实验方法的基 础上增加一个广角探测器, 扩大了散射角 $2 \theta$ 的探测 范围, 从而可以实现从几个埃到数百纳米范围内结 构的表征. 一般来说, 对于一维的广角探测器样品置 于探测器的中心点位置上效果最佳, 但是对于许多 时间分辨的 SAXS 实验通常需要引入拉伸仪、温度控 制台、剪切热台等原位实验装置, 由于受仪器空间以 及广角探测器的最大探测角度的限制, 广角探测器 的位置需要随原位实验装置的变化而变化, 如图 1 所 示为上海光源 BL16 的实验站仪器分布图, 广角弧形 探测器中心可以在距离样品中心 0-0.6 m 范围内自由 摆放, 因而能够引入各种原位实验装置. 而由于探测 器中心的偏离, 使得广角探测器所探测的角度不是 真实的散射角. 为了准确获得真实的散射角, 各个实验 站提出了不同的角度校正方法, 如台湾的 $\mathrm{BL} 17 \mathrm{~B} 3^{[15]}$
实验站根据其线形探测器的特点建立了一个关于探 测器像素通道和真实散射角的方程(校正方程), 然后 通过标准样品中的已知峰位求解方程来获得模型中 参数, 最后将模型参数代入校正方程来获得真实的 散射角. 而对于二维广角面探测器, 通常采用与小角 二维面探测器相同的校正方法, 需要根据标准样品 中的已知峰来获取样品到探测器中心的距离 $D$, 然后 通过三角正切关系求取真实的散射角, 校正的步骤 如图 2 中实线所示. 然而这两种方法都与标准峰位的 选取相关, 选择不同的几组标准峰就可能获得不同 的校正结果, 因而存在一定的随机性. 而且上述理论 模型中并未考虑到线形探测器偏离入射平面, 二维 面探测器与入射光并不严格垂直, 以及由于样品不 平整时和光斑发散等产生的角度偏差.

为解决上述问题, 本文以一维弧形探测器为基 础提出了一种构造校正方程的方法, 此方法同样适 用于一维线形探测器和二维面探测器的 WAXS 探测 器, 首先根据探测器和实验装置的关系构造出几何 模型, 然后对所建立的几何模型进行泰勒近似获得 校正方程，通过标准样品峰位拟合出校正方程的参 数，从而获得了更加简单、高效、稳定的校正方程，可 以实现 SWAXS 实验方法中不同维度探测器的广角 数据校正, 校正流程为图 3 虚线所示. 最后, 选用了 三组标准样品对校正方法进行了验证，为这一方法 的推广提供了理论和实验的支持.

\section{WAXS 校正方程}

\section{1 几何模型}

SWAXS 实验方法通常会引入一些原位的实验 装置, 使得探测器的中心往往偏离样品的中心. 如图

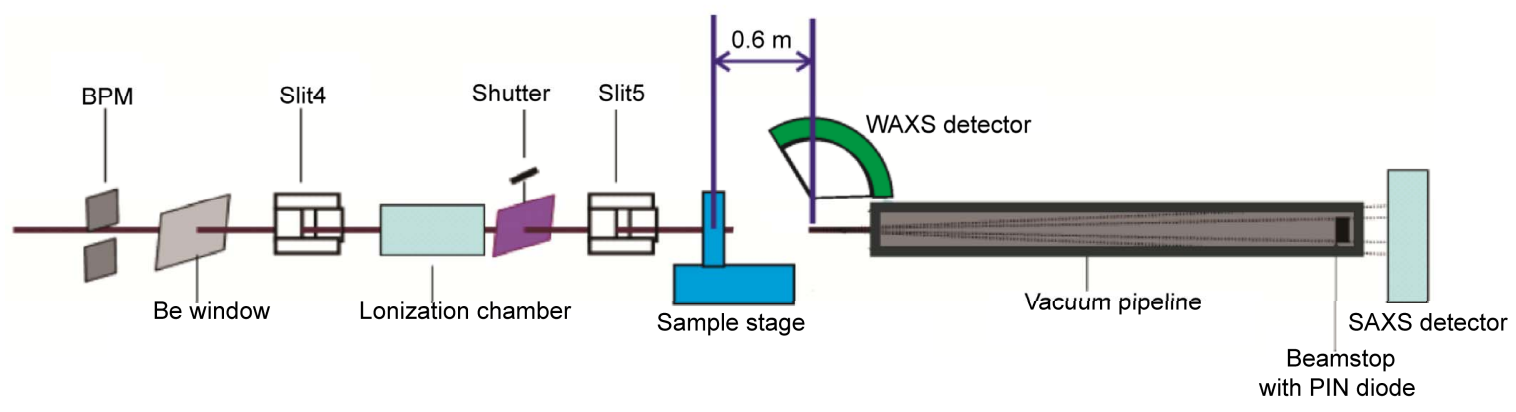

图 1 (网络版彩图) BL16B 光束线站的仪器分布图, WAXS 探测器离样品中心的最大距离为 $0.6 \mathrm{~m}$

Figure 1 (Color online) Layout of beamline BL16B, the maximum distance between sample and WAXS detector is $0.6 \mathrm{~m}$. 


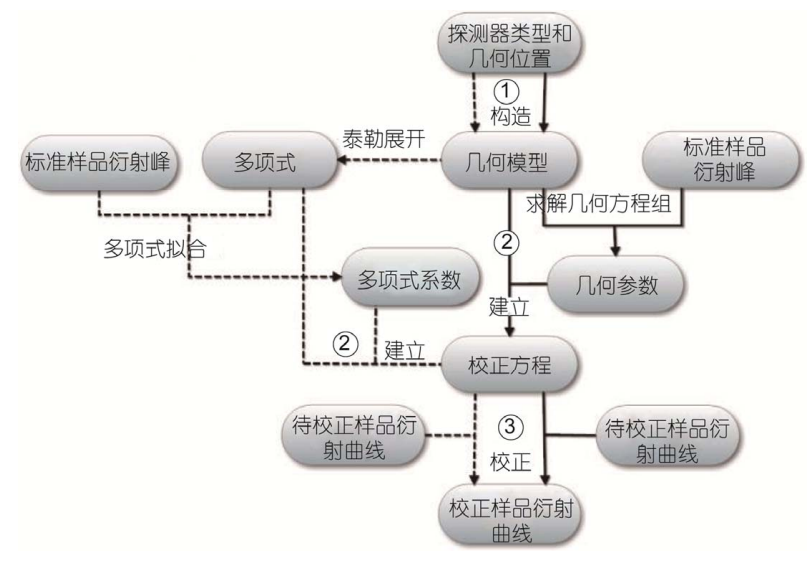

图 2 WAXS 数据校正的基本流程, 其中实线表示台湾 BL17B3 线站所使用的校正方法, 虚线表示本文所使用的 校正方法

Figure 2 The flow chart of calibrating WAXS data. Solid line represents the calibrating method used by beamline BL17B at Taiwan, dotted line represents the calibrating method used in the study.

3 所示, 以样品中心 $\mathrm{S}$ 为坐标原点, 建立直角坐标系 1 , 弧形探测器中心 $\mathrm{C}$ 的坐标为 $\left(x_{\mathrm{c}}, y_{\mathrm{c}}, z_{\mathrm{c}}\right)$, 其中散射 角 $2 \theta$ 为入射光束与散射光束的夹角. 在实际测量中, 探测器的所获得探测值为 $2 \theta_{\mathrm{d}}$, 当探测器中心与样品 中心重合时, 探测值 $2 \theta_{\mathrm{d}}$ 与真实散射角 $2 \theta$ 相等, 这在 原位实验中通常不能满足. 因此, 需要建立一个关联 探测值 $2 \theta_{\mathrm{d}}$ 与真实散射角 $2 \theta$ 的校正方程. 如图 3 所示, 以 $\mathrm{C}$ 为坐标原点建立直角坐标系 2 , 散射光束的探测 器接收点 $\mathrm{D}$ 在 2 坐标系的坐标为 $\left(x_{\mathrm{d} 2}, y_{\mathrm{d} 2}, z_{\mathrm{d} 2}\right)$, 设二维 弧形探测器平面与 $y_{2}, z_{2}$ 轴的夹角分别为 $\omega, \varphi$, 因此 D 在 2 坐标系下的坐标值可以通过旋转探测器平面内 $\mathrm{D}$ 的坐标 $\left(X_{\mathrm{d}}, Y_{\mathrm{d}}, Z_{\mathrm{d}}\right)$ 计算到

$$
\begin{aligned}
&\left(\begin{array}{l}
x_{d 2} \\
y_{d 2} \\
z_{d 2} \\
1
\end{array}\right)=\left(\begin{array}{cccc}
\cos (\varphi) \cos (\omega) & -\sin (\omega) & -\cos (\omega) \sin (\varphi) & 0 \\
\cos (\varphi) \sin (\omega) & \cos (\omega) & -\sin (\omega) \sin (\varphi) & 0 \\
\sin (\varphi) & 0 & \cos (\varphi) & 0 \\
0 & 0 & 0 & 1
\end{array}\right) \\
& \times\left(\begin{array}{l}
x_{d} \\
y_{d} \\
z_{d} \\
1
\end{array}\right), \\
& x_{d}=0 ; y_{d}=R \cdot \cos \left(2 \theta_{d}+\theta_{0}\right) ; z_{d}=R \cdot \sin \left(2 \theta_{d}+\theta_{0}\right) \cdot(1)
\end{aligned}
$$

其中 $R$ 为弧形探测器的半径. 在 1 坐标系下, $\mathrm{D}$ 的坐 标可由 2 坐标系平移得到

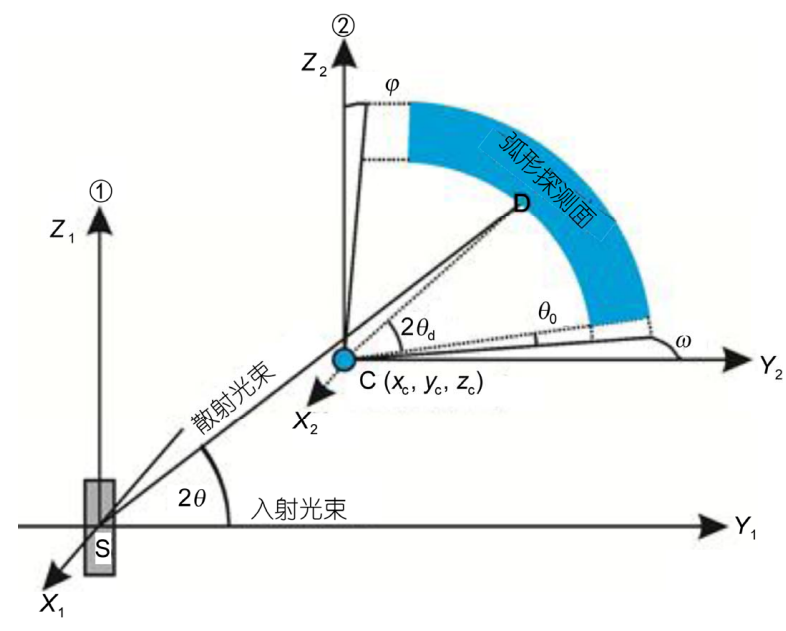

图 3 (网络版彩图) WAXS 散射角度校正的几何模型, $\mathrm{S}$ 为 样品中心, $C$ 为探测器中心, $2 \theta$ 为真实的散射角, $2 \theta_{\mathrm{d}}$ 为探测 器获得的散射角度, $\theta_{0}$ 为探测器的零点位置在(2)坐标系中 的角度, $\omega$ 为探测器平面与 $Y_{2}$ 轴的夹角, $\varphi$ 为探测器平面与 $Z_{2}$ 轴的夹角

Figure 3 (Color online) The geometry model for calibrating scattering angle in WAXS experiment. S represents the centre of sample, $\mathrm{C}$ represents the centre of detector, $2 \theta$ is real scattering angle, $2 \theta_{\mathrm{d}}$ is scattering angle obtained by detector, $\theta_{0}$ is the angle of zero point of WAXS detector in coordinate system (2), $\omega$ is the angle between detector plane and $Y_{2}$ axis, $\varphi$ is the angle between detector plane and $\mathrm{Z}_{2}$ axis.

$$
\begin{aligned}
& x_{d 1}=x_{D 2}+x_{c}, \\
& y_{d 1}=y_{d 2}+y_{c}, \\
& z_{d 1}=z_{d 2}+z_{c} .
\end{aligned}
$$

结合公式(1)与(2), 可以建立真实散射角 $2 \theta$ 与探 测值 $2 \theta_{\mathrm{d}}$ 的函数关系 $(3)$

$$
\begin{aligned}
& \cos (2 \theta)=\frac{y_{d 1}}{\sqrt{x_{d 1}{ }^{2}+y_{d 1}{ }^{2}+z_{d 1}{ }^{2}}}, \\
& x_{d 1}=x_{c}-R \cos \left(2 \theta_{d}+\theta_{0}\right) \sin (\varphi) \\
& -R \sin \left(2 \theta_{d}+\theta_{0}\right) \cos (\varphi) \sin (\omega) \text {, } \\
& y_{d 1}=y_{c}+R \cos \left(2 \theta_{d}+\theta_{0}\right) \cos (\varphi) \\
& -R \sin \left(2 \theta_{d}+\theta_{0}\right) \sin (\varphi) \sin (\omega), \\
& z_{d 1}=z_{C}+R \sin \left(2 \theta_{d}+\theta_{0}\right) \sin (\omega) \text {. }
\end{aligned}
$$

为了获得校正方程, 需要求解上述函数中的 6 个 参数, 由于是非线性的超越方程, 通常是选择一种或 一系列标样的若干衍射峰建立方程组, 然后用非线 性拟合的方法或智能算法寻找最优解. 因此, 解的准 确性与初始值的选择密切相关, 从而不能保证较为 稳定的解. 为了简化校正过程，对(3)进行泰勒展开, 
可得真实散射角 $2 \theta$ 与探测值 $2 \theta_{\mathrm{d}}$ 的多项式关系

$$
\begin{aligned}
2 \theta= & A+B \cdot\left(2 \theta_{d}\right)+C \cdot\left(2 \theta_{d}\right)^{2}+D \cdot\left(2 \theta_{d}\right)^{3} \\
& +R_{n+1}\left(2 \theta_{d}\right) .
\end{aligned}
$$

其中 $R_{n+1}\left(2 \theta_{\mathrm{d}}\right)$ 用于近似计算的误差估计. 在实际应用 中, (3) 方程中的 6 个参数的范围可以确定, 根据 BL16B 实验条件, 取 $x_{\mathrm{c}}=(-1-1 \mathrm{~cm}), y_{\mathrm{c}}=(0-60 \mathrm{~cm}), z_{\mathrm{C}}$ $=(0-60 \mathrm{~cm}), \theta_{0}=(0-10 \mathrm{deg}), \omega=(-2-2 \mathrm{deg}), \varphi=(-2-2$ $\mathrm{deg})$, 将参数代入 $R_{n+1}\left(2 \theta_{\mathrm{d}}\right)$ 中, 令 $\left|R n+1\left(2 \theta_{\mathrm{d}}\right)\right| \leqslant M$, 即 可求得在误差范围 $M$ 内, 所需要展开的多项式级数 $n$. 在本文中, $M$ 取国际上常用标准 0.1 度, 由于在原 位实验中 $\omega, \varphi$ 通常保持不变, 因此假定上述三参数为 不变量, 选定三组不同 $x_{\mathrm{c}}, y_{\mathrm{c}}, z_{\mathrm{c}}, \theta_{0}$ 实验条件(表 1)应 用公式(3)进行模拟计算(图 4), 模拟结果表明只有三 次拟合方程的标准残差小于 0.1 度. 因此, 校正方程 近似为四参数的三次多项式方程(公式(4)). 显然, 通 过坐标系变换建立几何模型以及结合泰勒近似来建 立校正方程方法也适应于其他类型的探测器, 如一 维线形探测器、二维面探测器等等. 为了验证模型和 近似方法的正确性，作者选用了 $\mathrm{LaB}_{6}, \mathrm{CeO}_{2}, \mathrm{Y}_{2} \mathrm{O}_{3}$ 三 组标准样品对理论模型和近似方法进行了验证, 获 得了较好的结果.

\section{2 实验}

本文根据探测器中心偏离样品中心的距离选定 了三组不同实验条件对三种标样分别进行测试, 这 三种模式的参数见表 1. 模式一的探测器偏离距离最 小, 但是存在一个较大的初始角度. 而模式二和模式 三的偏离距离相对模式一依次增大, 但是初始角度 较小. 受限于仪器的空间限制, 模式一和模式三分别 是可以达到的边界实验条件. 因此这三种模式可以 较为充分地验证模型的可行性范围.

校正实验选择的 $X$ 射线波长为 $0.124 \mathrm{~nm}$ ，探测 器为一维弧形探测器(法国 Inel 公司, CPS 120), 探测 范围为 0-120 度, 最小分辨角度为 0.014 度, 三种标 样的衍射角度均查自国际标准的 PDF 卡片库: $\mathrm{LaB}_{6}$ (34-0427), $\mathrm{CeO}_{2}$ (34-0349), $\mathrm{Y}_{2} \mathrm{O}_{3}$ (89-5592). 表 2 为三 种标准样品的标准散射角度.

\section{3 实验结果分析}

本文根据建立的几何模型(公式 3)验证了在不同 实验模式下探测器角度值 $2 \theta_{\mathrm{d}}$ 与真实散射角 $2 \theta$ 的三
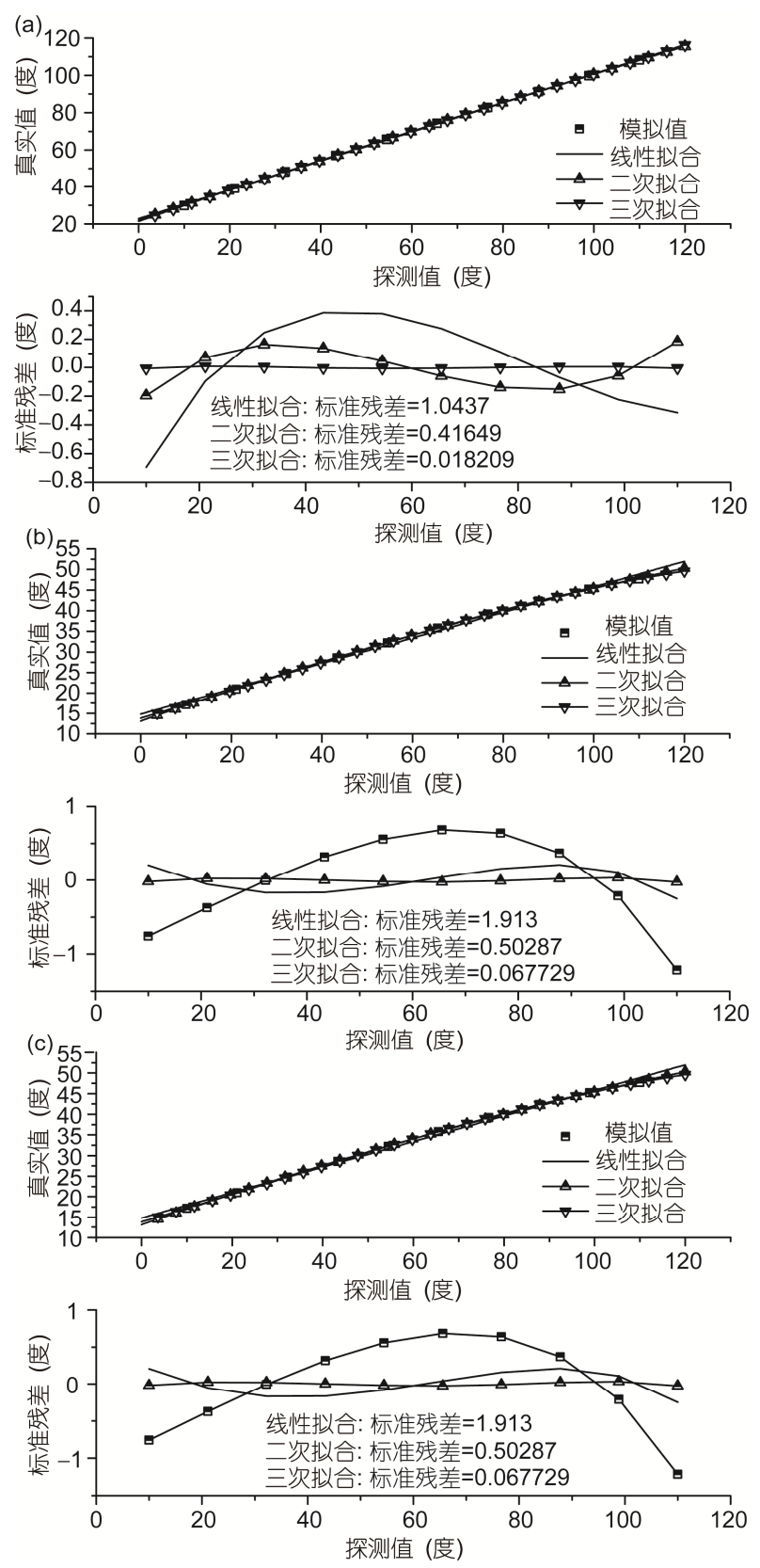

图 4 三组实验条件(表 1)的探测值与真实值的模拟结果

(a) $x_{\mathrm{c}}=0 \mathrm{~mm}, y_{\mathrm{c}}=5 \mathrm{~mm}, z_{\mathrm{c}}=77 \mathrm{~mm}, \theta_{0}=5 \mathrm{deg}$; (b) $x_{\mathrm{c}}=0 \mathrm{~mm}, y_{\mathrm{c}}=450 \mathrm{~mm}$, $z_{\mathrm{c}}=150 \mathrm{~mm}, \theta_{0}=3.61 \mathrm{deg}$; (c) $x_{\mathrm{c}}=0 \mathrm{~mm}, y_{\mathrm{c}}=470 \mathrm{~mm}, z_{\mathrm{c}}=130 \mathrm{~mm}$, $\theta_{0}=3.84 \mathrm{deg}$

Figure 4 The simulation results of scattering angle at three groups of experiment condition showed in table 1 . (a) $x_{\mathrm{c}}=0 \mathrm{~mm}, y_{\mathrm{c}}=5 \mathrm{~mm}, z_{\mathrm{c}}=77$ $\mathrm{mm}, \theta_{0}=5 \mathrm{deg}$; (b) $x_{\mathrm{c}}=0 \mathrm{~mm}, y_{\mathrm{c}}=450 \mathrm{~mm}, z_{\mathrm{c}}=150 \mathrm{~mm}, \theta_{0}=3.61 \mathrm{deg}$; (c) $x_{\mathrm{c}}=0 \mathrm{~mm}, y_{\mathrm{c}}=470 \mathrm{~mm}, z_{\mathrm{c}}=130 \mathrm{~mm}, \theta_{0}=3.84 \mathrm{deg}$.

次方关联, 由图 4 可知, 在设定的实验参数范围内, 只有三次多项式拟合可以满足角度校正所需的精度 
表 1 三组实验条件参数

Table 1 The parameters at three groups of experiment condition

\begin{tabular}{ccccc}
\hline 实验条件 & $\begin{array}{c}\text { 水平位置 } \\
x_{\mathrm{c}}(\mathrm{mm})\end{array}$ & $\begin{array}{c}\text { 水平位置 } \\
y_{\mathrm{c}}(\mathrm{mm})\end{array}$ & $\begin{array}{c}\text { 垂直位置 } \\
z_{\mathrm{c}}(\mathrm{mm})\end{array}$ & $\begin{array}{c}\text { 初始角度 } \\
\theta_{0}(\mathrm{deg})\end{array}$ \\
\hline 模式 1 & 0 & 5 & 77 & 5 \\
模式 2 & 0 & 450 & 155 & 3.61 \\
模式 3 & 10 & 470 & 130 & 3.84 \\
\hline
\end{tabular}

表 2 三种标样的标准峰位(度)

Table 2 The peak value of three kinds of standard sample

\begin{tabular}{cccc}
\hline 序号 & $\mathrm{LaB}_{6}$ & $\mathrm{CeO}_{2}$ & $\mathrm{Y}_{2} \mathrm{O}_{3}$ \\
\hline 1 & 24.353 & 22.902 & 16.474 \\
2 & 29.945 & 26.485 & 23.394 \\
3 & 34.721 & 37.818 & 27.069 \\
4 & 38.972 & 44.661 & 28.745 \\
5 & 42.862 & 46.768 & 31.852 \\
6 & 49.904 & 54.549 & 34.712 \\
7 & 53.904 & 59.916 & 38.644 \\
8 & 56.294 & 61.638 & 45.676 \\
9 & 59.3 & 68.267 & 46.768 \\
10 & 65.218 & 68.267 & - \\
11 & 67.066 & - & - \\
\hline
\end{tabular}

要求. 随着探测器原点偏离样品中心的距离的增大, 探测器所能探测到的真实散射角范围逐渐减小, 未 校正的广角 $X$ 射线散射曲线(图 5)中散射峰的个数随 偏心距离的增大而减少, 而散射峰的峰宽随着角度 的增大而宽化, 这是因为仪器在高角区域的视差效 应造成的.

为了验证几何模型和校正方法的可行性, 本文 通过将标样 $\mathrm{CeO}_{2}, \mathrm{Y}_{2} \mathrm{O}_{3}$ 的探测值与标准值代入校正 方程(4)进行拟合获得了校正参数, 然后根据所得的 校正参数对 $\mathrm{LaB}_{6}$ 标样的散射曲线进行角度校正. 如 图 6 所示为三种模式的校正曲线.

我们将 $\mathrm{LaB}_{6}$ 的校正结果与 PDF 卡片的标准值进 行了比较分析. 由表 3 的校正结果可知, 模式二与模 式三的校正结果与理论预测十分接近, 而模式一的校 正结果与理论存在一定的偏差, 这可能是由于模式一 中探测器的角度分辨要小, 而且视差效应在广角区域 更加明显, 从而使得峰的位置存在一定的位移. 另外, 峰位的标定方法也对校正结果产生较大的影响, 由于 在实验中, 选用的测试材料都是标准样品, 衍射峰位 的峰宽比较窄, 因此, 本实验使用的是 Origin 软件中 的阀值法和导数法来确定峰位, 但是对于有机材料, 需要根据峰的对称性, 选用合适的衍射峰形函数对衍 射峰进行精确的拟合，从而确定衍射峰的峰位.
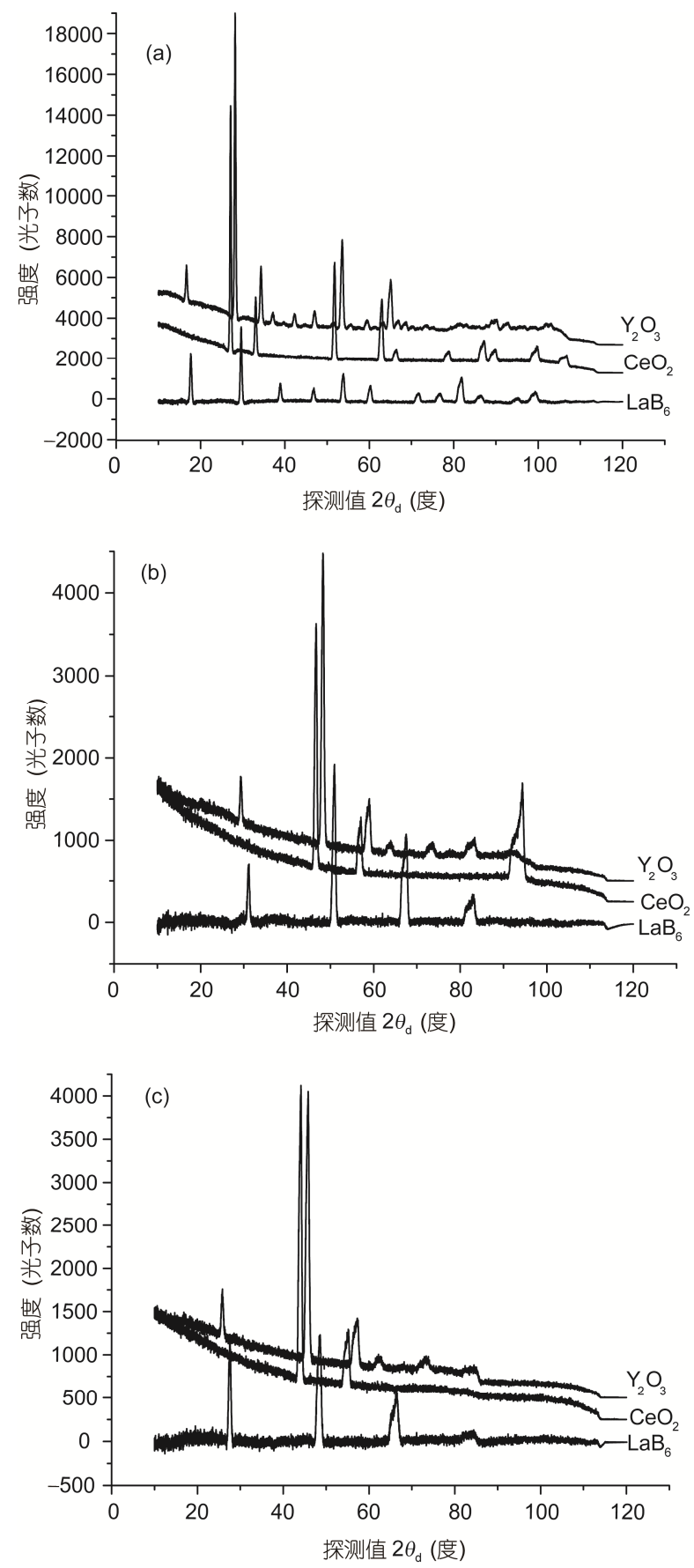

图 5 为标样在三组实验条件(表 1)下未校正角度的广角 $\mathbf{X}$ 射线散射曲线

Figure 5 The scattering curves of standard sample at three groups of experiment condition.

\section{4 结论}

同步辐射小角与广角联用的 X 射线散射方法通 

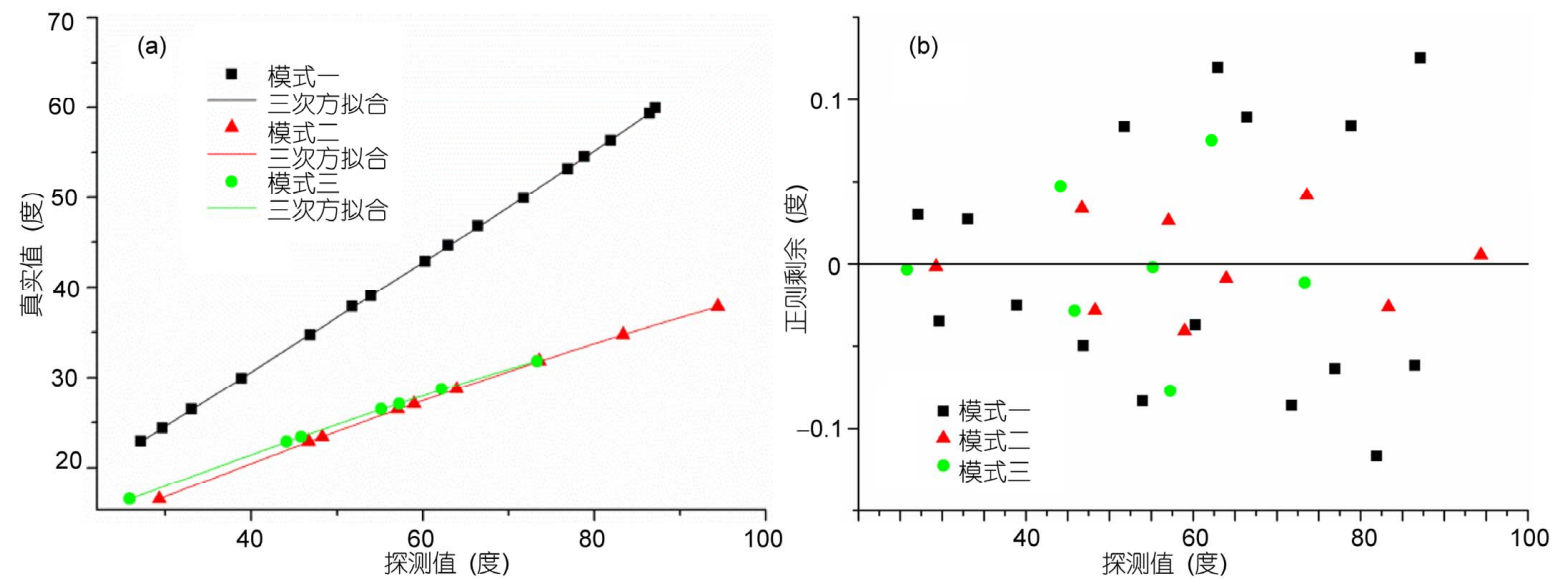

图 6 (网络版彩图)为三种模式的散射角度校正曲线. (a) 三次方拟合曲线; (b) 三次方拟合的正则剩余量

Figure 6 (Color online) The calibrating curve of three groups of experiment condition. (a) Fitting curve of cubic equation; (b) standard residuals of cubic fitting.

表3 $\mathrm{LaB}_{6}$ 在三种不同实验模式下的校正结果(单位: 度)

Table 3 The calibrating results of $\mathrm{LaB}_{6}$ at three groups of experiment condition

\begin{tabular}{|c|c|c|c|c|c|c|c|c|c|}
\hline \multirow{2}{*}{ 序号 } & \multicolumn{3}{|c|}{ 模式一 } & \multicolumn{3}{|c|}{ 模式二 } & \multicolumn{3}{|c|}{ 模式三 } \\
\hline & 标准值 & 校正值 & 相对误差 & 标准值 & 校正值 & 相对误差 & 标准值 & 校正值 & 相对误差 \\
\hline 1 & 16.474 & 16.496 & 0.001 & 17.152 & 17.19889 & 0.003 & 17.152 & 17.088 & 0.004 \\
\hline 2 & 23.394 & 23.520 & 0.005 & 24.353 & 24.36598 & 0.0006 & 24.353 & 24.356 & 0.0001 \\
\hline 3 & 27.069 & 27.226 & 0.005 & 29.945 & 29.9101 & 0.001 & 29.945 & 29.926 & 0.0006 \\
\hline 4 & 28.745 & 28.928 & 0.006 & 34.721 & 34.59828 & 0.003 & 34.721 & 34.643 & 0.002 \\
\hline 5 & 31.852 & 31.990 & 0.004 & - & - & - & - & - & - \\
\hline 6 & 34.712 & 34.877 & 0.005 & - & - & - & - & - & - \\
\hline 7 & 38.644 & 38.835 & 0.005 & - & - & - & - & - & - \\
\hline 8 & 45.676 & 45.838 & 0.004 & - & - & - & - & - & - \\
\hline 9 & 46.768 & 46.950 & 0.0039 & - & - & - & - & - & - \\
\hline 标准残差 & & 0.122 & & & 0.074 & & & 0.038 & \\
\hline
\end{tabular}

常需要对广角探测器的角度进行校正, 由于在原位 实验中需要引入各种原位设备, 广角探测器需要随 实验条件的改变而移动位置, 本文以上海光源 BL16 线站的实验条件为基础, 建立了用于校正原位实验
中广角探测器角度的通用方法. 应用几何方程可以 确定真实散射角的范围, 这有利于寻找标样的标准 峰位, 经过数学近似的校正方程大大简化了校正参 数的获得, 同时确保了校正结果的准确性与稳定性.

\section{参考文献}

1 朱育平. 小角 $X$ 射线散射一一理论、测试、计算及应用. 北京: 化学工业出版社, 2008. 1-3

2 Yan T Z, Zhao B J, Cong Y H, et al. Critical strain for Shish-Kebab formation. Macromolecules, 2010, 43(2): 602-605

3 Hu S S, Rieger J, Yi Z Y, et al. Structural evolution of a colloidal crystal fiber during heating and annealing studied by in situ synchrotron small angle X-ray scattering. Langmuir: ACS J Surf Colloids, 2010, 26(16): 13216-13220

4 Yan G Y, Tian Q, Huang C Q, et al. A small-angle X-ray scattering study of micro-defects in thermally treated HMX (in Chinese). Acta Physica Sin, 2012, 61(13): 136101 [阍冠云, 田强, 黄朝强, 等. 热损伤奥克托金(HMX)缺陷的 X 射线小角散射研究. 物理学报, 2012, 61(13): 136101] 
5 Sun G A, Chen B, Wu E D, et al. Small angle X-ray scattering study of the microstructure and interface characteristics of single crystal superalloys during creep process (in Chinese). Acta Physica Sin, 2011, 60(1): 016102 [孙光爱 陈波, 吴二冬, 等. 蠕变镍基单晶高温合 金微观结构与界面特征的 X 射线小角散射研究. 物理学报, 2011, 60(1): 016102]

6 Li L Q, Liu Y, Liu P, et al. Solution conformation of the response regulator proteins from Deinococcus radiodurans studied by SAXS. Chin Phys C, 2011, 35(10): 974-977

7 Rapp G, Gabriel A, Dosiere M, et al. A dual detector single readout system for simultaneous small-(saxs) and wide-angle x-ray (waxs) scattering. Nucl Instrum Methods Phys Res Sect A, 1995, 357(1): 178-182

8 Tsuruta H, Brennan S, Rek Z U, et al. A wide-bandpass multilayer monochromator for biological small-angle scattering and fiber diffraction studies. J Appl Crystallogr, 1998, 31: 672-682

9 Chu B, Hsiao B S. Small-angle X-ray scattering of polymers. Chem Rev, 2001, 101(6): 1727-1761

10 Narayanan T, Diat O, Bosecke P. SAXS and USAXS on the high brilliance beamline at the ESRF. Nucl Instrum Methods Phys Res Sect A, 2001, 467: 1005-1009

11 Riekel C. Applications of micro-SAXS/WAXS to study polymer fibers. Nucl Instrum Methods Phys Res Sect B, 2003, 199: 106-111

12 Bras W, Ryan A J. Sample environments and techniques combined with small angle X-ray scattering. Adv Colloid Interface Sci, 1998, 75(1): $1-43$

13 Amenitsch H, Bernstorff S, Laggner P. High-flux beamline for small-angle x-ray-scattering at elettra. Rev Sci Instrum, 1995, 66(2): $1624-1626$

14 Fujisawa T, Inoue K, Oka T, et al. Small-angle X-ray scattering station at the SPring-8 RIKEN beamline. J Appl Crystallogr, 2000, 33(1): $797-800$

15 Lai Y H, Sun Y S, Jeng U S, et al. An instrument for time-resolved and anomalous simultaneous small- and wide-angle X-ray scattering (SWAXS) at NSRRC. J Appl Crystallogr, 2006, 39(6): 871-877

16 Liu D G, Chang C H, Liu C Y, et al. A dedicated small-angle X-ray scattering beamline with a superconducting wiggler source at the NSRRC. J Synchrot Radiat, 2009, 16: 97-104

17 Paris $\mathrm{O}$, Li C H, Siegel S, et al. A new experimental station for simultaneous X-ray microbeam scanning for small- and wide-angle scattering and fluorescence at BESSY II. J Appl Crystallogr, 2007, 40: S466-S470

18 Krywka C, Sternemann C, Paulus M, et al. The small-angle and wide-angle X-ray scattering set-up at beamline BL9 of DELTA. J Synchrotron Radiat, 2007, 14(Pt 3): 244-251

19 Daniels J E, Pontoni D, Hoo R P, et al. Simultaneous small- and wide-angle scattering at high X-ray energies. J Synchrotron Radiat, 2010 , 17(4): 473-478

20 Li X, Tian F, Yang C, et al. In situ small angle X-ray scattering study on structural evolution of crosslinked polytetrafluoroethylene during deformation. J Appl Polym Sci, 2014, 131(4): 39883 


\title{
Calibrating scattering angle in wide angle $X$-ray scattering experiment
}

\author{
ZHAO Nie, BIAN FengGang, WANG YuZhu, YANG ChunMing, \\ ZHOU Ping \& WANG Jie*
}

ShangHai Synchrontron Radiation Facility, ShangHai Institute of Applied Physics, Chinese Academy of Sciences, Shanghai 201204, China

Impact by in-situ experiment device there are some discrepancies between scattering angle obtained from detector and the real scattering angle of the sample within simultaneous small and wide angle X-ray scattering experiment. Therefor method for calibrating scattering angle should be developed in various experiment conditions. Two problems must be solved in calibrating process: first, a calibration equation should be developed which include the main factors leading to angle deviation. The second problem is finding the corresponding real scattering angle of standard sample to scattering angle obtained from detector in different experiment modes. In this paper, method for building calibration equation was proposed on the base of one-dimensional arc detector. A geometry model was created in rectangular coordinates of the sample. Then the model was simplified by Taylor approximation according to required precision. Through numerical simulating of different experimental conditions, the validity of calibration equation was proved, and the simulating results could be used to solve the problem of finding corresponding relationship of scattering angle from detector with real scattering angle of the sample. Three standard samples were selected to verify the precision of calibration equation within different experimental conditions. The results show that mode 2 and 3 were consistent with the theory prediction. However in mode 1 the calibration precision was slightly larger than theory simulation. It may be caused by decreased angle resolution and parallax effect in experiment of mode 3 which was related to detector properties, beam divergence and the uneven sample. This method could be also used to build calibration equation for other type detectors, such as one-dimensional line detector or two dimensional surface detector.

synchrotron radiation, $X$-ray scattering, wide angle, angle calibration

PACS: 06.20.fb, 07.85.Qe, 78.70.Ck, 07.05.Tp

doi: $10.1360 /$ SSPMA2014-00106 